\section{Development and validation of an in vivo model for pharmacokinetic/pharmacody- namic studies of antimicrobials in domestic animals}

\author{
Maria Laura Meneses, ${ }^{1}$ Gabriela \\ Albarellos, 2 Maria Fabiana Landoni ${ }^{1}$ \\ 1Departamento de Ciencias Preclinicas, \\ Facultad de Ciencias Veterinarias, \\ Universidad Nacional de La Plata; \\ 2Departamento de Farmacología, \\ Facultad de Ciencias Veterinarias, \\ Universidad de Buenos Aires, Argentina
}

\section{Abstract}

The increase in bacterial resistance to antimicrobials has motivated researchers to develop experimental animal models to integrate pharmacokinetic/pharmacodynamic (PK/ PD) profiles to predict the effectiveness of antimicrobials treatments. The models used to date have a weakness based on the use of the minimum inhibitory concentration (MIC), whose value is obtained only in vitro, determined under constant conditions with an exponential error, regarding to the double dilution used. The aim of this study was to develop and validate a device for subcutaneous implantation, which can be applied in any animal species, allowing simultaneous description of in vivo bacterial killing curve and pharmacokinetic profile of the drug under study. Based on the obtained results, it can be concluded that the use of this model will allow researchers to apply PK/ PD decreasing the error originated in the use of MIC as a measure of antimicrobial pharmacodynamics.

\section{Introduction}

In the last decades, the emerging growth of bacterial resistance to antimicrobials has been studied extensively. Overuse and/or irrational use of antimicrobials favoured the selection of resistant microorganisms; this resistance may extend to other microbial populations, threatening humans and animals, including those not previously exposed to antimicrobial agents. ${ }^{1,2}$ In many bacterial infections, the goal of the antimicrobial therapy is not only to ensure clinical cure but to also achieve bacterial eradication (bacteriological cure). ${ }^{3}$ In this context, Toutain et al. ${ }^{4}$ emphasize the so-called Pollyanna phenomenon, which refers to the lack of correlation between clinical cure and bacteriological cure and the consequences of assessing antimicrobial efficacy in symptomatic responses exclusively.

The effect of an antimicrobial treatment is the result of a dynamic three-way interaction between the host, the drug, and the infecting organism. ${ }^{5}$

Pharmacokinetics describes the links between the drug and the host, while pharmacodynamics describes the links between the drug and the bacteria. It is important to highlight that a proper pharmacokinetic/pharmacodynamic (PK/PD) study should include a third component, the interaction between the host and the pathogen. ${ }^{4}$

The three PK/PD indices used as efficacy predictors relates different aspects of the concentration-time curve to the minimum inhibitory concentration (MIC): time (T>MIC), peak of the curve (Cmax/MIC), and the integrate of time and concentration calculated as area under the curve (AUC/MIC) ${ }^{6,7}$ All of these indices use MIC for correlating pharmacokinetics with potency. Although MIC is the simplest and most widely used measure of the intrinsic activity of antimicrobials, its use in the present situation is questionable.

Minimum inhibitory concentrations are generally determined by use of two-fold dilutions of the antimicrobial drug. The true MIC is mostly not the figure determined but is in between the observed MIC and the next lower dilution. The inherent error is one dilution step in either direction. ${ }^{8}$ Evidently, this imprecision may have a profound influence for the calculated PK/PD indices. On the other hand, in an infectious disease, there is a population of bacteria involved and not individual bacteria; therefore, it is important to know the distribution of the antimicrobial susceptibility of this population. ${ }^{9}$ Also by using MIC as pharmacodynamic parameter for evaluation of antimicrobial potency, any information related to the interaction between host and bacteria is unknown and impossible to be established. ${ }^{9}$

In the last years a number of experimental models for PK/PD studies have been reported; some of them are in vitro models, such as, the kinetic models of dilution or diffusion that simulate the clearance of drug from the system. ${ }^{10,11}$ There are also models of specific diseases that use fibrin clots or glass beads to simulate endocarditis or devices related infections, such as the one reported by McGrath $e t$ $a l .12$ These models are informative since they allow the description of the effect of defined concentration-time profiles of a drug on selected bacterial strains in a controlled and reproducible environment. ${ }^{3}$ Different aspects of PK/PD relationships, including selection of resistance, can be studied and have critical factors identified. These models are good tools for studies on most aspects of PK/PD relationships of antimicrobials. However, they are
Correspondence: Maria Fabiana Landoni, Departamento de Ciencias Preclinicas, Facultad de Ciencias Veterinarias, Avenida 60 y 118, cc 296 1900 La Plata, Buenos Aires, Argentina.

E-mail: landoni@fcv.unlp.edu.ar

Key words: antimicrobials, in vivo model, PK/PD studies, cephalexin.

Conflict of interests: the authors report no conflict of interests.

Received for publication: 6 October 2011.

Revision received: 16 February 2012

Accepted for publication: 20 February 2012.

This work is licensed under a Creative Commons Attribution NonCommercial 3.0 License (CC BYNC 3.0).

(C) Copyright M.L. Meneses et al., 2012

Licensee PAGEPress srl, Italy

Veterinary Science Development 2012; 2:e10 doi:10.4081/vsd.2012.e10

affected by the same factors as MIC determinations (e.g. medium, growth phase of inoculum and its density), and do not account for effects of the interaction between the host and the infecting organism.

Another model used to study PK/PD is the $e x$ vivo infection model; this consists of subcutaneous implantation tissue cages in animals, which can be stimulated by irritants, such as carrageenan to obtain exudate. The experimental animals are treated with an antimicrobial, and the exudate samples obtained are confronted ex vivo with standardized bacterial cultures in order to quantify the antibacterial activity over an incubation period of 24 hours. This model allows the calculation of AUC/MIC (AUIC) 24 for bacteriostasis (no change in bacterial counts), bactericidal activity $(99.9 \%$ reduction in the number of bacteria), or total bacterial eradication. ${ }^{14}$ Like the in vitro models, ex vivo models do not include the host defense mechanisms.

Finally, different in vivo infection models have been designed; these models include experimental infections in small rodents such those reported by Zak and 0 'Reilly ${ }^{15}$ and Andes and Craig. 16 These models are either general (thigh infections, peritonitis-septicemia) or reflect more specific disease conditions (pneumonia, meningitis, endocarditis, pyelonephritis, abscesses, foreign-body infections etc). ${ }^{17}$ Each model has its own limitations; there are general factors that affect the conclusions, such as choice of bacterial strains, inoculum size, time between infection and treatment and dosing regimen. 15 The main weakness of these models is the difference in pharmacokinetics between small rodents and larger animals. This can be solved, to some extent, through dose 
fractionation or prolongation of the elimination rate through, e.g., renal impairment. Also, the animals are often rendered neutropenic before infection, which nullifies the effect of the host defences. The present study presents a novel in vivo model. This model is designed to allow the bacteria growing and the transfer of free drug (not bound to plasma proteins) from the vascular space into the device and vice versa but prevents bacteria from leaking. In this article, the model is described and validated using a timedependent antimicrobial administered intravenously with two administration designs, emulating differing therapeutic situations (a unique high dose and lower doses with short dose intervals).

\section{Materials and Methods}

\section{Model Development}

Bacterial strain and inoculum preparation

Escherichia coli ATCC25922, was used in all experiments. It was kept at $-20^{\circ} \mathrm{C}$ in a tryptic soy broth with glycerol as cryoprotectant. Immediately prior to start of each experiment, an inoculum was prepared from a culture grown for 18h on Mueller Hinton agar. The culture was suspended in PBS to an approximate concentration of $1 \times 10^{8} \mathrm{cfu} / \mathrm{mL}(0.5 \mathrm{McF}$ arland scale).

Preparation of subcutaneous implanted devices (SID)

The devices consisted of polypropylene capsules of $12 \mathrm{~mm}$ of diameter by $10 \mathrm{~mm}$ of depth, with a $9 \mathrm{~mm}$ gap (Figure 1). A total of thirty-six SID were used. Thirty-two were filled with $100 \mu \mathrm{L}$ of $E$. coli inoculum diluted on $200 \mu \mathrm{L}$ of Brain Heart broth, to a final concentration of $1 \times 10^{5}$. Once filled, the gap was closed with a $0.24 \mu \mathrm{m}$ Millipore membrane adhere to the gap with methyl cyanoacrylate and reinforced with polypropylene rings sealed by hot melt.

The remaining four SID (not inoculated) were filled in, with $300 \mu \mathrm{L}$ of PBS/Brain Hearth broth (33/66), and used to evaluate both tissue reactions at the implantation site and the effect of incubation on the physicochemical characteristics of the content.

\section{Experimental animals}

Six 45-day-old male New Zealand White rabbits (3.0 to $3.5 \mathrm{~kg}$ each) were used.

The animals were kept in cages of $50 \mathrm{~cm}$ $\times 50 \mathrm{~cm} \times 60 \mathrm{~cm}$ and fed with a commercial diet (Gepsa Feeds, Pilar Group, RabbitHome Type). Food and water were given ad libitum. All animal procedures were approved by the Institutional Animal Care and Use Committee, School of Veterinary, University of La Plata, Argentina.

Subcutaneous devices implantation in rabbits/incubation at $37^{\circ} \mathrm{C}$

$E$. coli inoculated SID were split. Sixteen were placed in to test tubes containing $5 \mathrm{~mL}$ of brain heart broth and taken to an incubator at $37^{\circ} \mathrm{C}$. The remaining 16 were surgically implanted in 4 rabbits under anaesthesia (ketamine $25 \mathrm{mg} / \mathrm{kg}$ and xylazine $1 \mathrm{mg} / \mathrm{kg}$ ). Analgesia was assured by the intramuscular administration of buprenorphine at a dose rate of $0.05 \mathrm{mg} / \mathrm{kg}$. Two SID were prepared as described but, filled with PBS/Mueller Hinton. These devices were implanted in 2 rabbits and considered as negative controls. The day before the study, the dorsal hair of all the rabbits was cut with scissors and, shortly before the start of the study, shaved. The implantation site (dorsal line) was disinfected with povidone iodine, and four subcutaneous pockets were created. One SID was placed into each subcutaneous pocket. Afterwards, the incisions were sutured with nylon. At this time (recorded as time 0), SIDs into test tubes con- taining heart broth were taken to incubator at $37^{\circ} \mathrm{C}$.

\section{Extraction of subcutaneous implanted devices}

Devices were aseptically removed from the site of implantation or incubator, simultaneously, at 12, 24, 48 and 72 hours post-implantation/incubation. For the extraction of the implanted SID, rabbits were sedated with acepromazine at a dose rate of $1 \mathrm{mg} / \mathrm{kg}$ IM and regional nerves blockade (0.1-0.2 mL of $2 \%$ lidocaine at each site, Lidocaine ${ }^{\circledR}$, Richmond Vet Pharma, Argentina) for analgesia. At each sample time, after removing SID, swabs of the subcutaneous pocket were taken to check the proper closing of the device. The extracted SID were opened with sterile forceps and scissors, and $100 \mathrm{uL}$ of transudate was recovered. Transudate bacterial number was estimated by dilution and plating technique and expressed in $\mathrm{cfu} / \mathrm{mL}$.

The SID filled with PBS/MuellerHinton broth were extracted at 24 and 72 hours postimplantation, and a biopsy of the subcutaneous pocket tissue was sent to the Laboratory of Pathology, of the School of Veterinary Sciences of the National University of La Plata for histopathological studies. Physicochemical and biological features of the recovered liquid were evaluated by classical methods. ${ }^{18}$

\section{Model validation}

In order to validate the model, the antimicrobial effect of cephalexin (a $\beta$-lactam with time-dependent killing kinetics) administered intravenously using a single dose and a repeated dose schedule was studied. A total of 96SID were prepared as previously explained but alternatively used Staphylococcus aureus ATCC 25923 as test strain. Immediately prior to start of each experiment, an inoculum was prepared from a culture grown for 18 hours on Mueller

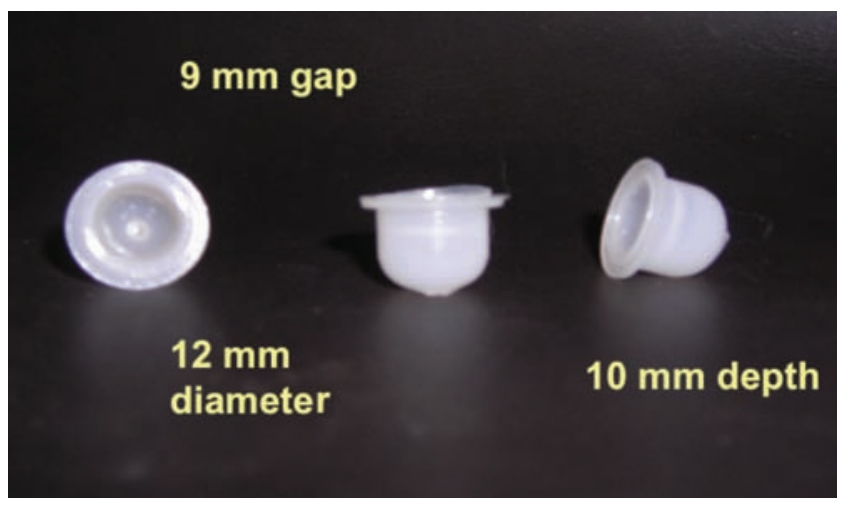

Figure 1. Subcutaneous implanted device.

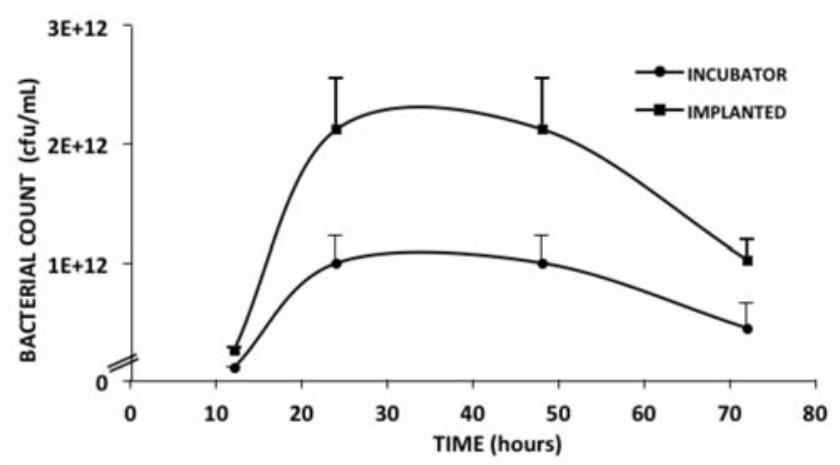

Figure 2. Time profile of bacterial growth $(\mathrm{cfu} / \mathrm{mL})$ into devices subcutaneously implanted in rabbit $(\cdot)$ and incubated at $37^{\circ} \mathrm{C}$ $(\bullet)$ during a 72 hours period. 
Hinton agar. The culture was suspended in isotonic saline solution to an approximate concentration of $1 \times 10^{8} \mathrm{cfu} / \mathrm{mL}(0.5$ McFarland), and then it was re-diluted to yield a final inoculum of approximately $1 \times 10^{5} \mathrm{cfu} / \mathrm{mL}$. Sixteen 45-day-old male New Zealand White rabbits (3.2 to $3.8 \mathrm{~kg}$ ) were used. Devices were surgically implanted (six in each animal) as previously described. Twenty-four hours after surgery, animals were divided into four groups, as follows:

- Group $1(\mathrm{n}=4)$ received cephalexin intravenously at a dose rate $10 \mathrm{mg} / \mathrm{kg}$, registering the end of administration as 0 time.

- Group $2(n=4)$ received a total of four 2.5 $\mathrm{mg} / \mathrm{kg}$ intravenous doses of cephalexin every 6 hours.

- Groups $3(n=4)$ and $4(n=4)$ received no antimicrobial treatment and were used as control for groups 1 and 2 , respectively.

One of the implanted devices and a blood sample were withdrawn from groups 1 and 3 at $3,6,9,12,18$ and 24 hours post-administration and at $0.5,1,3,6,9$ and 24 hours post-administration for the remaining groups.

Blood samples were withdrawn through a 24 G (Abbocat) catheter placed in the marginal ear vein. Devices were removed and processed as previously described.

Cephalexin plasma concentrations were quantified by microbiological method using Microccocus luteus ATCC 9341 as a microorganism test. 19

The procedures for viable bacteria counts were as described previously.

Individual cephalexin concentration $v s$. time curves were analyzed by non-linear least square regression analysis using PCNonlin (SCI Software, $4^{\text {th }}$ Edition, 1992, Lexington, USA). Initial estimates were determined using the residual method 20 and refitted by non linear regression. The number of exponents needed was determined by applying the Schwartz and Akaike criterions ${ }^{21,22}$ and the residual distribution around the estimated concentrations. Most pharmacokinetic parameters were calculated using classic equations associated with compartmental analysis. ${ }^{20}$

\section{Results}

Physicochemical characteristics of the content recovered from non-inoculated SID shown similarities to transudate: low protein content, low specific gravity $(<1.012)$, and absence of nucleated cells.

Histopathological studies of implant area did not show signs of local inflammation. All cultures from swabs collected from subcutaneous pockets showed no bacterial growth indicating a proper closure of the SID.

Bacterial growth curves in both rabbit
implanted-SID and incubated-SID showed a proper and comparable bacterial growth. The peak growth was observed at $24 \mathrm{~h}$ in both the implanted and incubated-SID (Figure 2). No statistically significant differences were observed when areas under the curve (AUC) $\mathrm{cfu} / \mathrm{mL}$ vs time from incubated and implanted SID were compared $(\mathrm{P}=0.27)$.

Cephalexin plasma concentration curves and bacterial growth curves from implanted SID for both administration schedules are presented in Figures 3 and 4. Cephalexin plasma concentration vs time curves after single and repeated intravenous administration were best fitted to an open monocompartmental model in all the animals; the following equation was used:

$$
\mathrm{C}_{(\mathrm{t})}=\mathrm{C}_{\mathrm{p}} \exp (-\mathrm{K} . \mathrm{t})
$$

where $C_{(t)}(\mu \mathrm{g} / \mathrm{mL})$ represents cephalexin plasma concentration at $\mathrm{t}$ time; $\mathrm{C}_{\mathrm{p}}(\mu \mathrm{g} / \mathrm{mL})$ is the concentration extrapolated to time 0 of the first phase of cephalexin plasma disposition and $\mathrm{K}$ (per h) is the elimination slope.

The average equation for the model was:

$$
\text { Monodose } \quad \mathrm{Cp}=5.87 \mathrm{e}^{-0.06 \mathrm{t}}
$$$$
\text { Repeated dose } \quad \mathrm{Cp}=1.63 \mathrm{e}^{-0.08 \mathrm{t}}
$$

Estimated pharmacokinetic parameters are shown in Table 1.

After intravenous administration of

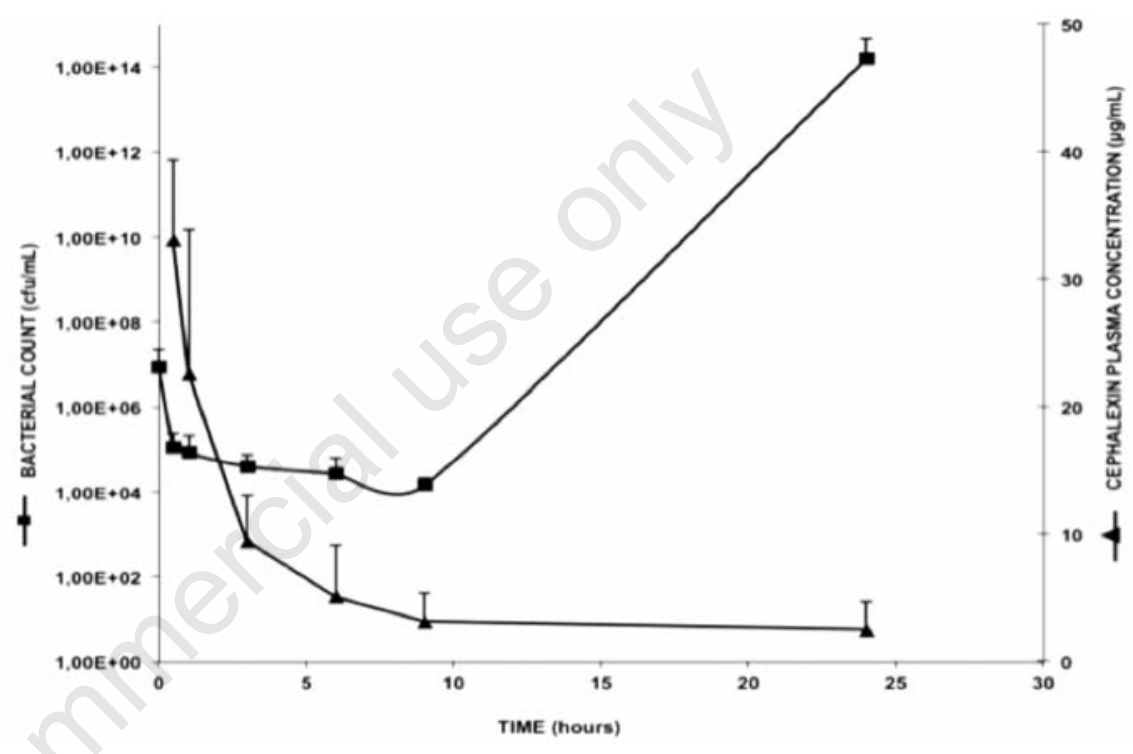

Figure 3. Mean and SD cephalexin plasma concentration $(\Delta)$ and bacterial growth into SID ( $\square$ ) time profile after its single intravenous administration at a dose rate of $10 \mathrm{mg} / \mathrm{kg}$ to rabbits $(n=4)$.

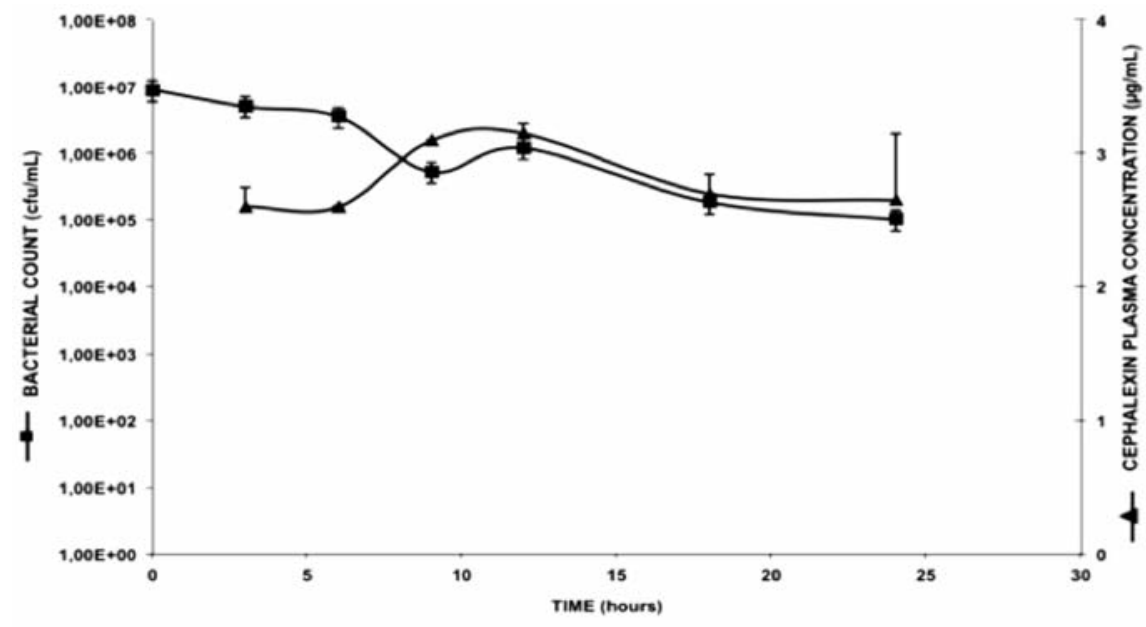

Figure 4. Mean and SD cephalexin plasma concentration $(\Delta)$ and bacterial growth into SID ( $\square$ ) time profile after its repeated intravenous administration at a dose rate of 2.5 $\mathrm{mg} / \mathrm{kg} / 6 \mathrm{~h}$ to rabbits $(\mathrm{n}=4)$. 
Table 1. Mean $( \pm$ SD) pharmacokinetic parameters of cephalexin after intravenous administration at a single dose of $10 \mathrm{mg} / \mathrm{kg}$ and repeated dose of $2.5 \mathrm{mg} / \mathrm{kg} / 6 \mathrm{~h}$ to rabbits.

\begin{tabular}{|c|c|c|c|c|}
\hline \multirow[b]{2}{*}{ PK parameter } & \multicolumn{2}{|c|}{$\begin{array}{l}\text { Single dose } \\
(10 \mathrm{mg} / \mathrm{kg})\end{array}$} & \multicolumn{2}{|c|}{$\begin{array}{l}\text { Repeated dose } \\
(2.5 \mathrm{mg} / \mathrm{kg} / 6 \mathrm{~h})\end{array}$} \\
\hline & Mean & SD & Mean & SD \\
\hline $\mathrm{K}(1 / \mathrm{h})$ & 0.06 & 0.01 & 0.08 & 0.01 \\
\hline $\operatorname{AUC}_{(0-\infty)}(\mu \mathrm{g} \cdot \mathrm{h} / \mathrm{mL})$ & 94.69 & 9.22 & 21.27 & 1.58 \\
\hline$t_{1} / 2(h)$ & 11.18 & 1.41 & 9.04 & 1.08 \\
\hline $\mathrm{C}_{\mathrm{p}(0)}(\mu \mathrm{g} / \mathrm{mL})$ & 5.87 & 0.28 & 1.63 & 0.09 \\
\hline $\mathrm{Cl}_{\mathrm{B}}$ (L/h.kg) & 0.11 & 0.01 & 0.12 & 0.01 \\
\hline Vd (l/kg) & 1.70 & 0.08 & 1.53 & 0.09 \\
\hline
\end{tabular}

$\operatorname{AUC}_{(0-\infty)}$, area under the plasma concentration vs time curve from 0 to infinite; $\mathrm{C}_{\mathrm{p}(0)}$, plasma concentration at 0 time; $\mathrm{Vd}$, volume of distribution; $\mathrm{Cl}_{\mathrm{B}}$, body clearance; $\mathrm{K}$, elimination rate constant; $\mathrm{t} / 2$, elimination half-life.

cephalexin following a monodose schedule bacterial counts curves had very low inter-animal variation. Bacterial growth curves had a similar time profile in all experimental animals, with a 2 log drop in bacterial counts at early times followed by a significant re-growing at 24 hours post-administration. This profile fits very well to the cephalexin plasma concentration $v s$ time (Figure 3).

In the multi-dose intravenous administration schedule, bacterial growth curves were similar for all the experimental rabbits; however, variation was slightly higher than that recorded for the single dose study. Bacterial count drop more slowly than after single dose administration, in concordance to time required for cephalexin plasma concentration to achieve a steady state. Once steady state is achieved bacterial count dropped two log, without re-growth through the end of the study (Figure 4).

\section{Discussion}

In the last two decades, a number of experimental in vivo models for studies of dose/effect relationships of antimicrobials have been reported. ${ }^{6}$ All of these have advantages and disadvantages, with outcomes that should be interpreted accordingly. ${ }^{15,23} \mathrm{~A}$ model for studies on PK/PD relationships should, ideally, enable a detailed description of the pharmacokinetics of the drug, the pharmacodynamic effect on bacteria, and preferably also of the host contribution to the measured effects, allowing for the calculation of optimal doses and rational dosing schedules.5,24,25

The model described here allows for repeated sampling and quantification of the effect of any antimicrobial in presence of host-defence system. Compared to the tissue cage models, such as, the ones described by Clarke ${ }^{26}$ and Greko, ${ }^{27}$ the present model does not induce any systemic sign of infection. This may be due to the proper closure system of the device, where there was no local tissue reaction on the implantation zone. Therefore, the safety of the experimental animals is not affected.

The developed model has shown robustness for differentiating the efficacy of different dosing schedules. As for the single-dose studies, they showed that bacterial growth decreased when cephalexin concentration was high in the early hours of the trial. After 12 hours, the fall of cephalexin plasma concentrations lead to an increase of bacterial growth, which exceeded the initial inoculum.

Moreover, the administration of cephalexin at a dose rate of $2.5 \mathrm{mg} / \mathrm{kg}$ every 6 hours inhibited bacteria growing slower than after the single dose. This is probably related to the time required for cephalexin plasma concentrations to reach steady state. Once this is accomplished, the bacterial count dropped from $10^{7}$ to $10^{5} \mathrm{cfu} / \mathrm{mL}$, without increasing. It is important to highlight that at the end of the study eradication was not observed; this is somewhat unexpected as cephalexin, being a betalactam, is considered time dependent bactericidal. ${ }^{28}$ An explanation for this unexpected result could be the presence of biofilm inside the device, which has a much higher MIC than the bacteria in planktonic state. Although, the Staphylococcus strain used in the present study starts growing as biofilm after 48 hours of implantation (unpublished observation). A second explanation could be related to the presence of a number of bacteria on stationary phase. These bacteria are defined as persisters. ${ }^{29}$ Persisters represent a small subpopulation of cells that spontaneously enter a dormant, non dividing state. When a population is treated with a bactericidal antibiotic, regular cells die, whereas persisters survive. In order to kill, betalactams require active targets, which could explain the observed tolerance.

To conclude, it can be stated that the application of this model will enable interested researchers to describe in vivo effects of antimicrobials and to apply PK/PD model. This model will be applicable for studying any antibiotic, against any bacteria (planktonic or biofilm) in any animal species, leading to the design and/or the selection of rational and individual dosing regimens.

\section{References}

1. Toutain PL. Pharmacokinetics/pharmacodynamics integration in dosage regimen optimisation for veterinary medicine. J Vet Pharmacol Therap 2003;26:1-8.

2. Toutain PL. Antibiotic treatment of animals-a different approach to rational dosing. Vet J 2003;165:98-100.

3. Dagan R, Klugma, KP, Craig, WA, Baquero F. Evidence to support the rationale that bacterial eradication in respiratory tract infection is an important aim of antimicrobial therapy. J Antimicrob Chemother 2001;47:129-40.

4. Toutain PL. Pharmacokinetics/pharmacodynamics integration in drug development and dosage regimen optimisation for veterinary medicine. AAPS J 2002;4:38.

5. Drusano GL. Antimicrobial pharmacodynamics: critical interactions of 'bug and drug'. Nature Rev Microbiol 2004;2:289300.

6. Mouton JW, Dudley, MN, Cars 0, et al. Standardization of pharmacokinetic/pharmacodynamic (PK/PD) terminology for anti-infective drugs. Int $\mathrm{J}$ Antimicrob Agents 2002;19:355-8.

7. Toutain PL, Lees P. Integration and modelling of pharmacokinetic and pharmacodynamic data to optimise dosage regimens in veterinary medicine. J Vet Pharmacol Therap 2004;27:467-77.

8. Mueller M, de la Pena A, Derendorf $\mathrm{H}$. Issues in pharmacokinetics and pharmacodynamics of antiinfective agents: kill curves versus MIC. Antimicrob Agents Chemother 2004;48:369-77.

9. Udekwu K, Parrish N, Ankomah P, et al. Functional relationship between bacterial cell density and the efficacy of antibiotics J Antimicrob Chemother 2009;63:745-57.

10. Toothaker RD, Welling PG, Craig WA. An in vitro model for the study of antibacterial dosage regimen design. J Pharm Sci 1982; 71:861-4.

11. Koritz G, Kilroy C, Bevill R. Pharmacokinetics/pharmacodynamic modelling of antibacterial therapy in vitro. Proceedings 6th International Congress EAVPT 1994. No23

12. McGrath BJ, Kang G, Kaatz, GW. Teicoplanin, vancomycin, and gentamicin bactericidal activity alone and in combination against Staphylococcus aureus in an in vitro pharmacodynamic model of infective endocarditis. Antimicrob Agents Chemother 1994;38:2034-40.

13. Vogelman B, Gudmundsson S, Leggett J, et al. Correlation of antimicrobial pharmacokinetic parameters with therapeutic efficacy in an animal model. $\mathrm{J}$ Infect Dis 1988;158:831-47 
14. AliAbadi F, Lees P.Rational dosing of antimicrobial drugs: animals versus humans. Int J Antimicrob Agents 2002;19: 269-84.

15. Zak 0, O'Reilly T. Animal models in the evaluation of antimicrobial agents. Antimicrob Agents Chemother 1991;35: 1527-31.

16. Andes D, Craig WA. Animal models in the evaluation of antimicrobial agents. Antimicrob Agents Chemother 2002;35: 1527-31.

17. Leggett J E, Ebert S, Fantin B, Craig WA. Comparative dose-effect relations at several dosing intervals for betalactam, aminoglycoside and quinolone antibiotics against gram-negative bacilli in murine thigh-infection and pneumonitis models. Scand J Infect Dis 1991;74Suppl1:179-84.

18. Gázquez J, Porcel M, Vives M. Comparative analysis of Light's criteria and other biochemical parameters for distinguishing transudates from exudates. Resp Med
1998;92:762-5.

19. Bennet J, Brodie JL, Bennet EJ. Simplified accurate method for antibiotic assay of clinical specimens. Appl Microbiol 1966; 14:170-7.

20. Gibaldi M, Perrier D. Pharmacokinetics, 2nd ed. New York: Marcel Dekker Inc; 1982.

21. Schwartz G. Estimating the dimension of a model. Ann Stat1978;6:461-4.

22. Yamaoka K, Nakagawa T, Uno T. Application of Akaike's information criterion (AIC) in the evaluation of linear pharmacokinetic equations. J Pharmacokinet Biopharm 1978;6:165-75.

23. Andes D, Craig WA. Animal model pharmacokinetics and pharmacodynamics: a critical review. Int $\mathrm{J}$ Antimicrob Agents 2002;19:261-8.

24. Guyonnet J, Monnoyer S, Manco B, et al. In vivo pharmacokinetics and in vitro pharmacodynamics as a basis for predicting dosage of colistin in piglet g.i.t. disease. J
Vet Pharmacol Therap 2003;26Suppl1:1489.

25. AliAbadi F, Lees P. Rationalising dosage regimens of antimicrobial drugs; a pharmacological perspective. J Med Microbiol 2000:49:943-5.

26. Clarke CR. Tissue-chamber modelling systems:/applications in veterinary medicine. J Vet Pharmacol Ther 1989;12:349-68.

27. Greko C, Finn M, O'hagen P, et al. A tissue cage model in calves for studies on pharmacokinetic/pharmacodynamic interactions of antimicrobials. Int J Antimicrob Agents 2003;22:429-43.

28. Ambrose PG, Craig WA, Bhavnani SM, Dudley M. Pharmacodynamic comparisons of different dosing regimens of penicillin G against penicillin-susceptible and resistant pneumococci. Proceedings 42nd ICAAC 2002; Abstract A-635.

29. Lewis K. Persister cells. Ann Rev Microbiol 2010;64:357-72. 